\title{
Can relative rotation angle serve as a sensitive predictor for the development of adjacent segment degeneration after single-segment PLIF?
}

\author{
Xiao Liu \\ Peking University Third Hospital \\ Haonan Liu \\ Bayi Children's Hospital \\ Xiaosong Yang \\ Peking university \\ Zhongjun Liu \\ Peking University Third Hospital \\ xiaoguang liu ( $\sim 165221980 @ q q . c o m$ ) \\ Peking University Third Hospital \\ Miao Yu \\ Peking University Third Hospital
}

\section{Research article}

Keywords: adjacent segment disease, posterior lumbar interbody fusion, radiography, surface topography, comparison

Posted Date: March 20th, 2020

DOI: https://doi.org/10.21203/rs.3.rs-18055/v1

License: (1) (1) This work is licensed under a Creative Commons Attribution 4.0 International License. Read Full License 


\section{Abstract}

Background: Adjacent segment degeneration (ASD) is a major issue after posterior lumbar interbody fusion (PLIF). Several studies have reported the potential causes of ASD based on radiography. However, the postoperative dynamic changes in the adjacent segments are not clear. This study aimed to determine the effect of PLIF on ASD using a formetric 4D system and to compare the effectiveness of this system with that of traditional radiography for the prediction of ASD.

Methods: Eighty-five consecutive patients who underwent PLIF of a single-segmen t were included. The formetric 4Dsystem was used to calculate the relative rotation angle between the fusion segment and the upper and lower adjacent vertebrae preoperatively and at 6, 12 and 24 months postoperatively. The range of motion (ROM) and disc height (DH) of the adjacent segments were measured using radiography preoperatively and at 24 months postoperatively. At the final follow-up, the visual analogue scale (VAS) and Oswestry disability index (ODI) were used to evaluate the surgical outcome. The patients were divided into two groups according to the occurrence of radiographic ASD: the ASD group with progression of degeneration and the N-ASD group without progression of degeneration. The clinical outcomes and measurement data between the two groups were compared and analyzed.

Results: The index fusion segments included L2-3 to L5-S1. Preoperatively, the relative rotation angles between the fused segment and the upper and lower adjacent vertebrae were $5.1^{\circ} \pm 2.2^{\circ}$ and $3.3^{\circ} \pm 2.0^{\circ}$,respectively, and both angles increased significantly at all time-points after surgery $(p<0.05)$. The angles changed most significantly during L2-3 fusion. Radiographic ASD was noted in 13 of 85 patients $(15.3 \%)$ at 24 months. There was no significant difference in the DH, ROM, or clinical outcome between the two groups $(p>0.05)$, while the relative rotation angle with the upper adjacent vertebra was greater in the ASD group than in the N-ASD group $(p<0.05)$.

Conclusion: The relative rotation angle with the adjacent vertebra increased significantly after lumbar fusion surgery. It may be a more sensitive predictor for the development ofradiographic ASD than flexion-extension ROM and DH.

\section{Introduction}

During the past few decades, posterior lumbar interbody fusion (PLIF) has been widely used for treating degenerative lumbar disease, and satisfactory results have been reported in many studies [1]. Unfortunately, it alters the normal biomechanics of the spine as a result of decompression and fusion and creates a compensatory increase of motion at the unfused segments [2]. Therefore, the prevalence of pathological conditions after lumbar fusion such, as adjacent segment degeneration (ASD), has increased in recent years. The most common manifestation of ASD includes the degeneration of the intervertebral discs, segmental instability, facet joint hyperplasia, and spinal stenosis [2-5]. After the onset of ASD, patients may be at a higher risk of reoperation subsequently. At present, ASD is a common postoperative complication in patients with lumbar degenerative diseases [2].

Although there are many studies on the causes of ASD after fusion, the specific mechanisms remains controversial [4-6]. In a review, Hashimoto et al. [4] reported that ASD is related to factors, such as age, body mass index (BMI), genetic factors, preoperative degeneration of the adjacent segments, excessive distraction of the fused level, multi-segment fusion, and insufficient lumbar lordosis. Yugue et al. [7] found that the incidence of ASD increased significantly after lumbar fusion in patients with pre-existing spinal stenosis of the adjacent segments, greater facet tropism, and high BMI. Moreover, Omair et al. [6] found that polymorphism of the IL18RAP gene may be associated with the decrease in the adjacent disc's height, although the precise mechanism is unclear.

Recently, several authors conducted studies on ASD using imaging technology; however, most of these studies evaluated degeneration using lumbar spine imaging tests (e.g., radiography or magnetic resonance imagining) in a specific position [8,9]. To analyze the dynamic changes in the adjacent segments after lumbar fusion and their effect on the development of ASD, we implemented a formetric 4D system (DIERS, International GmbH of Schlangenbad, Germany; hereafter referred to as DIERS) to evaluate the patients who underwent singlesegment PLIF.

\section{Materials And Methods}

\section{Inclusion and exclusion criteria}

Patients aged $<65$ years with degenerative lumbar diseases, including lumbar disc herniation and/or lumbar spinal stenosis who underwent single-segment PLIF from January 2016 to September 2016 were enrolled in this study. We excluded patients with significant degeneration (University of California at Los Angeles grading scale classification $\geq$ Grade II, Table 1) or instability of the adjacent segments ${ }^{[10]}$, those complicated with spinal deformity or tumor, those with cervical spondylosis and/or thoracic spondylosis, those with walking difficulties, those with severe obesity or scar(s) substantially affecting stripe projection, those with a previous history of spinal surgery, those with failure 
of fusion as noted on radiographs or instrument failure postoperatively. This study was approved by the ethics committee of Peking University Third Hospital (M2018098). All patients involved in the study provided informed consent.

Table 1 University of California at Los Angeles grading scale for lumbar degeneration

\begin{tabular}{|c|c|c|c|}
\hline Grade & Disc-space narrowing & Osteophytes & End Plate sclerosis \\
\hline ૫ & $\square$ & $\square$ & $\square$ \\
\hline ? & $\square$ & $\square$ & $\square$ \\
\hline प & $\nabla / \mathbb{Q}$ & [ & [ \\
\hline प & $\nabla / \mathbb{\nabla}$ & $\nabla / \square$ & ] \\
\hline
\end{tabular}

“ $\nabla$ ” means yes; “ $\nabla ”$ means no

\section{Intervention}

Selective PLIF was performed for the segments that corresponded to the clinical manifestations and imaging examination findings. All surgeries were performed by senior surgeons.

\section{Application of DIERS system and radiographic evaluation}

The DIERS system was used to collect data on the rotation angles of the fusion segment with the upper and lower adjacent vertebrae in the coronal plane during three gait cycles. Subsequently, the difference between the rotation angles (i.e., relative rotation angles) formed by the fusion segment with the upper and lower adjacent vertebrae was calculated. The relative rotation angles were recorded preoperatively and periodically at 6,12 , and 24 months postoperatively.

The range of motion (ROM) and disc height (DH) of the adjacent segments were measured using lumbar X-ray preoperatively and at 24 months postoperatively. The diagnostic criteria for ASD on the radiographs were as follows: (1) DH decrease by >3 mm; (2) vertebral osteophyte formation $>3 \mathrm{~mm}$; (3) slippage of the adjacent vertebral body $>3 \mathrm{~mm}$; and (4) dynamic intervertebral space angulation $>5^{\circ}$ [1113]. All measurements were performed independently by two surgeons who were not involved with the procedures.

\section{Instructions for using DIERS}

\section{General instructions}

The DIERS system relies on the surface topography method to generate a three-dimensional model of the back. It does not involve radiation and can be applied without any damage or side-effects. The patients' anatomical landmarks, spinal curvature, and derived parameters can all be calculated based on the reconstructed anatomical models.

\section{Working principles}

First, the raster lines are evenly projected on the back of a standing patient to generate an optical image with a thick-and-thin alternation. The computer then generates a digital photograph of the back, which is coded in three colors, namely, red, green, and blue (for convex, saddle, and concave areas, respectively). The bony landmarks can be labeled on the back using special curvature changes, shown as isolated red areas surrounded by green areas. Finally, the computer recreates a three-dimensional spinal model using the collected surface topography data, and the software automatically continues to analyze the dynamic parameters related to spinal movements during walking.

\section{Specific operations}

For the measurement procedure, the patient must be undressed, except for the underpants, and the entire buttock area is uncovered. The patient is instructed to walk on the treadmill with a normal gait at a constant speed for $30 \mathrm{~s}$, during which a dynamic three-dimensional spine 
model is generated by the DIERS system. The angle between the vertebral body and the coronal plane is automatically recorded from each frame, and then the relative rotation angle is calculated (Figure 1).

\section{Comparison of clinical outcomes and measurements}

The visual analogue scale (VAS) and Oswestry disability index (ODI) were used to evaluate the patient outcomes at 24 months postoperatively. The patients were divided into two groups according to the occurrence of ASD: the ASD group with progression of degeneration and the N-ASD group without progression of degeneration. Both the clinical outcome and radiographic measurements of the two groups were compared (Figure 2).

\section{Statistical analysis}

SPSS version 21.0 (SPSS, Chicago, IL, USA) was used for statistical analysis. The results were expressed as means and standard deviations (SD). The Shapiro-Wilk (W) test was performed to verify the normal distribution of the measured data. Student's $t$ test was used when the measured data were normally distributed, otherwise the Wilcoxon $T$ test was used. The relative rotation angles between the fused segments and adjacent vertebrae at various time-points were analyzed using repeated measure analysis of variance. $P$-values less than 0.05 were considered statistically significant.

\section{Results}

\section{Baseline characteristics}

A total of 105 patients were enrolled in this study, according to the inclusion and exclusion criteria. Of these, 20 patients withdrew from the study during follow-up. Finally, complete data were obtained from 85 (81.0\%) patients. This study group had a mean age of 45.2 years (SD 11.3) and $42.4 \%$ were female. The index segments were L2-3 in 6 cases, L3-4 in 10 cases, L4-5 in 39 cases, and L5-S1 in 30 cases. The preoperative VAS scores for the lower back and leg pain were 3.2 points (SD 1.3) and 6.4 points (SD 2.0), respectively, and the ODI index was $65.3 \%$ (SD 12.5).

\section{Relative rotation angles}

As shown in Table 2, the relative rotation angles formed by the fused segment with the upper and lower adjacent vertebrae preoperatively were $5.1^{\circ}(\mathrm{SD} 2.2)$ and $3.3^{\circ}$ (SD 2.0), respectively. The relative rotation angles at all postoperative time-points were significantly greater than those recorded preoperatively $(p<0.05)$. Of these, the angles at 12 months were significantly greater than those at 6 months $(p<0.05)$. In contrast, no significant changes were found in the relative rotation angles at the later time-points $(p>0.05)$. At the final follow-up, the relative rotation angles of the fused segment with the upper adjacent vertebra were greater than those of fused segment with the lower adjacent vertebra $(p<0.001)$. The differences in the relative rotation angles between the fused segment and the upper and lower adjacent vertebrae measured postoperatively compared with the angles recorded preoperatively were $3.7^{\circ}(\mathrm{SD} 2.9)$ and $2.7^{\circ}$ (SD 2.5 ), respectively, and the change in the upper adjacent vertebra was significant $(p=0.033)$. As shown in Table 3 , the relative rotation angles were greater if the index segments were fused at higher levels. The relative rotation angles with the adjacent vertebrae (L1, L4) were the largest when L2-3 were fused. 
Table2 Relative rotation angle of adjacent segments before and after surgery (unit: degree)

\begin{tabular}{lll} 
& Upper adjacent segment & Lower adjacent segment \\
\hline Before surgery & $5.1 \pm 2.2$ & $3.3 \pm 2.0$ \\
\hline At 6 months & $7.5 \pm 3.3^{\mathrm{a}}$ & $4.7 \pm 1.8^{\mathrm{a}}$ \\
\hline At 12 months & $8.9 \pm 4.2^{\mathrm{ab}}$ & $5.9 \pm 2.9^{\mathrm{ab}}$ \\
\hline At 24 months & $8.8 \pm 4.6^{\mathrm{abc}}$ & $6.0 \pm 3.2^{\mathrm{abc}}$ \\
\hline
\end{tabular}

${ }^{a} \mathrm{P}$ value is less than 0.05 when the data compared with that before surgery

${ }^{\mathrm{b}} \mathrm{P}$ value is less than 0.05 when the data compared with that at 6 months

${ }^{\mathrm{c}} \mathrm{P}$ value is greater than 0.05 when the data compared with that at 12 months

Table 3 Relative rotation angle of upper and lower adjacent segments after fusion (unit: degree)

$\begin{array}{lllll}\text { Fused segment } & \text { L2-3 } & \text { L3-4 } & \text { L4-5 } & \text { L5-S1 } \\ \text { Upper adjacent segment } & 10.5 \pm 3.6(\mathrm{~L} 1) & 9.8 \pm 4.0(\mathrm{~L} 2) & 8.6 \pm 3.1(\mathrm{~L} 3) & 8.2 \pm 3.6(\mathrm{~L} 4) \\ \text { Lower adjacent segment } & 8.1 \pm 3.9(\mathrm{~L} 4) & 6.2 \pm 2.4(\mathrm{~L} 5) & 5.4 \pm 2.0(\mathrm{~S} 1) & -\end{array}$

L: lumbar vertebra; S: sacral vertebra

\section{Radiographic evaluation and clinical outcome}

As shown in Table 4, the ROM and DH of the adjacent segments did not change significantly at 24 months ( $p>0.05)$. Finally, radiographic ASD was identified in 13 patients (15.3\%), 7 of whom showed ASD in the higher levels (L1-4) and 6 showed ASD in the lower levels (L4-S1). ASD manifested as a decrease in the DH in 9 patients and as intervertebral space angulation $>5^{\circ}$ in 4 patients. None of the patients showed significant osteophytic formation or slippage of the vertebral body.

Table 4 Intervertebral range of motion and disc height of adjacent segments before and after surgery

\begin{tabular}{|c|c|c|c|c|c|c|c|c|}
\hline \multirow{2}{*}{$\begin{array}{l}\text { Fused } \\
\text { segment }\end{array}$} & \multicolumn{2}{|l|}{ L2-3 } & \multicolumn{2}{|l|}{ L3-4 } & \multicolumn{2}{|l|}{ L4-5 } & \multicolumn{2}{|l|}{ L5-S1 } \\
\hline & $\begin{array}{l}\text { Before } \\
\text { surgery }\end{array}$ & $\begin{array}{l}\text { At } 24 \\
\text { months }\end{array}$ & $\begin{array}{l}\text { Before } \\
\text { surgery }\end{array}$ & $\begin{array}{l}\text { At } 24 \\
\text { months }\end{array}$ & $\begin{array}{l}\text { Before } \\
\text { surgery }\end{array}$ & $\begin{array}{l}\text { At } 24 \\
\text { months }\end{array}$ & $\begin{array}{l}\text { Before } \\
\text { surgery }\end{array}$ & $\begin{array}{l}\text { At } 24 \\
\text { months }\end{array}$ \\
\hline $\begin{array}{l}\text { Upper } \\
\operatorname{ROM}\left({ }^{\circ}\right)\end{array}$ & $9.3 \pm 1.0$ & $10.5 \pm 0.4$ & $8.7 \pm 2.2$ & $9.6 \pm 1.3$ & $7.0 \pm 1.8$ & $7.7 \pm 2.1$ & $7.1 \pm 2.6$ & $7.7 \pm 2.3$ \\
\hline $\begin{array}{l}\text { Lower } \\
\text { ROM }\left(^{\circ}\right)\end{array}$ & $6.5 \pm 1.3$ & $7.2 \pm 2.0$ & $8.2 \pm 2.3$ & $8.7 \pm 2.3$ & $5.1 \pm 2.1$ & $5.0 \pm 1.9$ & - & - \\
\hline $\begin{array}{l}\text { Upper } \\
\text { DH } \\
(\mathrm{mm})\end{array}$ & $9.0 \pm 1.3$ & $8.2 \pm 1.0$ & $8.4 \pm 1.5$ & $7.7 \pm 1.7$ & $10.2 \pm 1.8$ & $9.5 \pm 1.6$ & $11.2 \pm 2.1$ & $10.7 \pm 1.6$ \\
\hline $\begin{array}{l}\text { Lower } \\
\text { DH } \\
(\mathrm{mm})\end{array}$ & $10.5 \pm 2.0$ & $9.8 \pm 1.6$ & $11.0 \pm 1.9$ & $10.5 \pm 2.1$ & $9.7 \pm 1.6$ & $9.1 \pm 1.4$ & - & - \\
\hline
\end{tabular}

The surgery was completed successfully in all patients. At 24 months, the VAS scores for the lower back and lower limb pain decreased to 1.3 points (SD 0.9) and 1.2 points (SD 0.7), respectively, and the ODI decreased to $10.6 \%$ (SD 5.9). Furthermore, no significant postoperative exacerbation of the neurological symptoms was reported. As reported in Table 5, there was no significant difference in the clinical outcome, $\mathrm{DH}$, and intervertebral ROM between the ASD group ( 13 cases) and N-ASD group ( 72 cases; $p>0.05$ ) at the final follow-up. In contrast, the relative rotation angles with the upper adjacent vertebrae in the ASD group increased significantly $(p=0.021)$. Notably, the significant change in the relative rotation angles was detected at as early as 6 months postoperatively (data not shown, $p<0.05$ ). 
Table 5 Comparison of clinical outcomes and measurement data between the two groups

\begin{tabular}{llll}
\hline & ASD group & N-ASD group & P value \\
\hline VAS (back pain) & $1.3 \pm 0.7$ & $1.3 \pm 1.0$ & 0.662 \\
\hline VAS (leg pain) & $1.3 \pm 1.0$ & $1.2 \pm 0.7$ & 0.701 \\
\hline ODI (\%) & $11.7 \pm 8.4$ & $10.4 \pm 4.3$ & 0.723 \\
\hline Upper ROM $\left(^{\circ}\right)$ & $9.3 \pm 3.1$ & $8.3 \pm 2.2$ & 0.182 \\
\hline Lower ROM $\left(^{\circ}\right)$ & $6.3 \pm 2.0$ & $6.9 \pm 2.4$ & 0.434 \\
\hline Upper DH $(\mathrm{mm})$ & $9.2 \pm 3.0$ & $9.9 \pm 2.4$ & 0.287 \\
\hline Lower DH $(\mathrm{mm})$ & $9.4 \pm 2.9$ & $10.1 \pm 3.0$ & 0.331 \\
\hline Relative rotation angle & & & 0.021 \\
\hline Upper adjacent segment $\left(^{\circ}\right)$ & $10.8 \pm 3.0$ & $8.6 \pm 2.8$ & 0.776 \\
\hline Lower adjacent segment $\left(^{\circ}\right)$ & $6.5 \pm 3.8$ & $5.9 \pm 2.0$ & \\
\hline
\end{tabular}

\section{Discussion}

ASD or adjacent segment disease (ASDis) is one of the most common postoperative complications after spinal fusion. The former refers to radiological changes at the segment adjacent to the fused spinal levels, and the latter is defined as degeneration of the adjacent segments that leads to clinical symptoms. The incidence of ASD varies from $5.6-100 \%$ in the available literature, which is higher than that of ASDis, whose incidence ranges from $5.2-18.5 \%[3,4,14]$. Presently, ASDis is considered one of the main causes of recurrent symptoms, and some patients have to undergo secondary surgery [15]. Therefore, we require a new method for detecting ASD in the light of the higher risk of developing ASDis.

Several studies have reported the prevalence and risk factors of ASD after lumbar spinal fusion; however, the specific mechanisms involved in the incidence of ASD remain controversial [5, 14-16]. Some authors believe that ASD is a natural process of degeneration that is not related to lumbar fusion [17]. However, several recent studies confirmed that segmental fusion may be the main cause of ASD [3, 18, 19]. Rao et al. [19] reported that the intradiscal pressure and motion at the adjacent segments increased significantly after single-segment lumbar fusion. In a prospective study of 111 patients with more than 10 years' follow-up, Ekman et al. [3] found that the incidence of ASD in surgical patients was significantly higher than that in patients receiving conservative treatment, suggesting that both fusion and laminectomy accelerate degeneration in the adjacent segments. Presently, most researchers believe that the development of ASD is related to factors, such as postoperative biomechanical changes, increased concentration stress in the intervertebral discs and facet joints, and hypermobility of the adjacent segments. However, the relevant evidence was derived primarily from the autoptic analysis, biomechanical studies, and finite element analysis $[3,19]$; these analyses can not reliably reproduce the postoperative changes in the adjacent during normal daily activities. Radiography was often used to evaluate the degenerative changes in the adjacent segments; however, it only provided static twodimensional projections rather than immediate and dynamic data. Therefore, we implemented the DIERS system to dynamically monitor the rotation angles of different vertebrae and to study the actual motion of the adjacent vertebrae after lumbar fusion. The advantages of the DIERS system include the following: (1) static measurements can be obtained without exposure to radiation; (2) actual vertebral rotation can be reproduced without considering the position of the pelvis; and (3) dynamic changes in the various vertebrae during walking, a major activity during routine life, can be indicated. The DIERS system analyzed the dynamic parameters related to the human body more accurately, and the measurements were reliable and reproducible, thereby, contributing to the study of the underlying mechanisms of ASD $[20,21]$.

Recently, several studies reported that the postoperative ROM of the adjacent segments was mostly increased-especially for the upper segment-while varying degree of decrease in the $\mathrm{DH}$ was noted. Berg et al. [22] conducted a 2-year follow-up of 72 patients after lumbar fusion and found that the ROM of the adjacent segments L3-4 and L4-5 increased by $4.3^{\circ}$ and $6.0^{\circ}$ compared with a decrease of $0.8^{\circ}$ in the ROM of L5-S1. In that study, the authors also observed that the postoperative DH decreased slightly. In the current study, we found similar changes in the ROM and DH of the adjacent segments at 2 years postoperatively, and the data did not differ significantly compared to the preoperative data. However, the difference between the previous studies and our study was that we firstly, to our knowledge, found the relative rotation angles with the adjacent vertebrae increased significantly after fusion. This suggests that although the overall stability of 
the lumbar spine does not change significantly after surgery, the range of rotation of the adjacent vertebrae inevitably increases to compensate for the lost function of the fused segments and to maintain the normal posture during normal activity. Notably, such changes occur earlier than the changes in the $\mathrm{DH}$, intervertebral ROM, or lumbar spinal stability. Consequently, we believe that the relative rotation angles between the fused segments and adjacent vertebrae measured by the DIERS system can detect the occurrence and development of ASD earlier than traditional radiography.

Various risk factors have been proposed regarding the occurrence of ASD [23]. To reduce the effect of the confounding factors, we only included patients aged less than 60 years who underwent single-segment fusion without significant degeneration of the adjacent segments. In our study, 13 patients developed ASD at the final follow-up and 9 patients showed decreased intervertebral spaces. By analyzing the preoperative radiographic images, we found that these 9 patients already had mild degeneration in the adjacent segments before the surgery. Therefore, we believe that the preoperative degeneration of the adjacent segments may be the most important factor leading to postoperative ASD. Another important factor affecting the development of ASD is the location of the fused segment. Many studies have reported that the fusion of the cranial segments is more likely to result in ASD development. Cheh et al. [2] found that the incidence of ASD after L1-3 fusion was higher than that after L4-5 fusion. Disch et al. [16] also demonstrated that patients who underwent fusion of the L4-5 segments showed significantly higher risks for ASD development than patients with fusion of the L5-S1 segments. In the current study, we found that the postoperative ROM and DH of the adjacent segments did not change significantly. However, the relative rotation angles with the adjacent vertebrae were greater if the index segments were fused at higher levels, and the change in the upper adjacent vertebra was more significant. The occurrence of such changes may be associatedwith the following factors: (1) the L4-S1 levels contribute almost $50 \%$ towards overall lumbar lordosis and provide the highest ROM for flexion/extension movements of the lumbar spine. After fusion, the upper adjacent levels compensate for the segmental immobility of L4-S1; (2) the L2-L4 levels are close to the thoracolumbar segments, which serve as transition zones for spinal curvature. If the upper lumbar segments were fused, the forces will be redistributed to the cranialadjacent segments because of the special biomechanical characteristics of the thoracolumbar region; and (3) placement of the pedicle screw can damage the superior facet, thereby, inevitably affecting the stability of the upper adjacent segments.

The development of ASD is a chronic process, and only a small number of patients experience clinical symptoms in the short term. Although the incidence of ASD is relatively high, few patients ultimately require surgery. Our results suggest that the clinical symptoms and lumbar spinal function significantly improved after 24 months for most patients. Even the 13 patients who developed radiographic ASD in the present study had clinical outcomes similar to those of their counterparts, suggesting that there was no significant correlation between radiographic ASD and the clinical outcomes in the short term. Notably, although the DH and intervertebral ROM for both groups were comparable at 24 months, the relative rotation angle of the upper adjacent vertebra in the ASD group was significantly greater. These results suggest that the overall structure of the lumbar spine remains stable in the resting state, regardless of the occurrence of ASD; nevertheless, excessive mobility of the adjacent segment persists during walking in the patients with ASD. Therefore, we believe that the relative movement between the adjacent and fused segment may be a more sensitive predictor of the development of ASD.

There are several limitations of our study. First, we only included cases with single-segment lumbar fusion, and patients with multilevel fusion were excluded. Second, the follow-up period of this study was only 2 years, and the long-term changes in the relative rotation angle after lumbar fusion must be investigated further. Finally, to reduce the effect of preoperative degeneration in the adjacent segments, the elderly patients were excluded from the study; this does not conform to the demographic characteristics. These issues will be addressed in the future.

\section{Conclusion}

PLIF is an effective surgery for patients with lumbar degenerative diseases. After fusion, the stability of the lumbar spine does not change significantly in the short term; however, the dynamic rotation movement of the adjacent segments is expected to increase, especially that of the upper adjacent segments. The rotation angles with adjacent segments may be more sensitive predictors for the development of ASD than flexion-extension ROM and DH.

\section{Abbreviations}

ASD

adjacent segment degeneration; PLIF:posterior lumbar interbody fusion; ROM:range of motion; DH:disc height; VAS:visual analogue scale; ODI:Oswestry disability index; BMI:body mass index; SD:standard deviations; ASDis:adjacent segment disease

\section{Declarations}


Not applicable.

\section{Authors' contributions}

$\mathrm{XL}$ and HNL conceived the research hypothesis and study design, collected the data, performed statistical analysis, and wrote the draft manuscript. ZJL, XGL and MY performed the PLIF surgery and assisted in project administration. XSY assisted in data collection and statistical analysis. XGL and MY edited the manuscript draft and made critical revisions. All authors read and approved the final manuscript.

\section{Funding}

This study was supported by Peking University Third Hospital (BYSYZD2019030).

\section{Availability of data and materials}

The datasets used and/or analyzed during the current study are available from the corresponding author on reasonable request.

\section{Ethics approval and consent to participate}

This study was approved by the ethics committee of Peking University Third Hospital (M2018098). All patients involved in the study provided informed consent.

\section{Consent for publication}

Not applicable.

\section{Competing interests}

The authors declare that they have no competing interests.

\section{Author details}

${ }^{1}$ Department of Orthopedics, Peking University Third Hospital, No. 49 North Garden Road, Haidian District, Beijing 100191, China

${ }^{2}$ Department of Orthopedics, Beijing Children's Hospital, Capital Medical University, National Center for Children's Health, 56 South Lishi Road, Xicheng District, Beijing 100045, China

\section{References}

1.

de Kunder SL, van Kuijk SMJ, Rijkers K, Caelers I, van Hemert WLW, de Bie RA, et al. Transforaminal lumbar interbody fusion (TLIF) versus posterior lumbar interbody fusion (PLIF) in lumbar spondylolisthesis: a systematic review and meta-analysis. Spine J. 2017;17(11):1712-21. 2. Cheh G, Bridwell KH, Lenke LG, Buchowski JM, Daubs MD, Kim Y, et al. Adjacent segment disease followinglumbar/thoracolumbar fusion with pedicle screw instrumentation: a minimum 5-year follow-up. Spine (Phila Pa 1976). 2007;32(20):2253-7. 3. 
Ekman P, Moller H, Shalabi A, Yu YX, Hedlund R. A prospective randomised study on the long-term effect of lumbar fusion on adjacent disc degeneration. Eur Spine J. 2009;18(8):1175-86.

4.

Hashimoto K, Aizawa T, Kanno H, Itoi E. Adjacent segment degeneration after fusion spinal surgery-a systematic review. Int Orthop. 2019;43(4):987-93.

5.

Pan A, Hai Y, Yang J, Zhou L, Chen X, Guo H. Adjacent segment degeneration after lumbar spinal fusion compared with motion-preservation procedures: a meta-analysis. Eur Spine J. 2016;25(5):1522-32.

6.

Omair A, Mannion AF, Holden M, Leivseth G, Fairbank J, Hagg O, et al. Age and pro-inflammatory gene polymorphisms influence adjacent segment disc degeneration more than fusion does in patients treated for chronic low back pain. Eur Spine J. 2016;25(1):2-13.

7.

Yugue I, Okada S, Masuda M, Ueta T, Maeda T, Shiba K. Risk factors for adjacent segment pathology requiring additional surgery after singlelevel spinal fusion: impact of pre-existing spinal stenosis demonstrated by preoperative myelography. Eur Spine J. 2016;25(5):1542-9.

8.

Jia YH, Sun PF. Preliminary evaluation of posterior dynamic lumbar stabilization in lumbar degenerative disease in Chinese patients. Chin Med J (Engl). 2012;125(2):253-6.

9.

Yu LP, Qian WW, Yin GY, Ren YX, Hu ZY. MRI assessment of lumbar intervertebral disc degeneration with lumbar degenerative disease using the Pfirrmann grading systems. PloS one. 2012;7(12):e48074.

10.

Ghiselli G, Wang JC, Hsu WK, Dawson EG. L5-S1 segment survivorship and clinical outcome analysis after L4-L5 isolated fusion. Spine (Phila Pa 1976). 2003;28(12):1275-80. discussion 80.

11.

Makino T, Honda H, Fujiwara H, Yoshikawa H, Yonenobu K, Kaito T. Low incidence of adjacent segment disease after posterior lumbar interbody fusion with minimum disc distraction: A preliminary report. Medicine. 2018;97(2):e9631.

12.

Chen BL, Wei FX, Ueyama K, Xie DH, Sannohe A, Liu SY. Adjacent segment degeneration after single-segment PLIF: the risk factor for degeneration and its impact on clinical outcomes. Eur Spine J. 2011;20(11):1946-50.

13.

Okuda S, Oda T, Miyauchi A, Tamura S, Hashimoto Y, Yamasaki S, et al. Lamina horizontalization and facet tropism as the risk factors for adjacent segment degeneration after PLIF. Spine (Phila Pa 1976). 2008;33(25):2754-8.

14.

Park P, Garton HJ, Gala VC, Hoff JT, McGillicuddy JE. Adjacent segment disease after lumbar or lumbosacral fusion: review of the literature. Spine (Phila Pa 1976). 2004;29(17):1938-44.

15.

Lee CS, Hwang CJ, Lee SW, Ahn YJ, Kim YT, Lee DH, et al. Risk factors for adjacent segment disease after lumbar fusion. Eur Spine J. 2009;18(11):1637-43.

16.

Disch AC, Schmoelz W, Matziolis G, Schneider SV, Knop C, Putzier M. Higher risk of adjacent segment degeneration after floating fusions: long-term outcome after low lumbar spine fusions. J Spinal Disord Tech. 2008;21(2):79-85.

17.

Battie MC, Videman T, Parent E. Lumbar disc degeneration: epidemiology and genetic influences. Spine (Phila Pa 1976). 2004;29(23):267990.

18.

Anandjiwala J, Seo JY, Ha KY, Oh IS, Shin DC. Adjacent segment degeneration after instrumented posterolateral lumbar fusion: a prospective cohort study with a minimum five-year follow-up. Eur Spine J. 2011;20(11):1951-60.

19.

Rao RD, David KS, Wang M. Biomechanical changes at adjacent segments following anterior lumbar interbody fusion using tapered cages. Spine (Phila Pa 1976). 2005;30(24):2772-6.

20.

Degenhardt B, Starks Z, Bhatia S, Franklin GA. Appraisal of the DIERS method for calculating postural measurements: an observational study. Scoliosis Spinal Disord. 2017;12:28.

Page $9 / 11$ 
21.

Frerich JM, Hertzler K, Knott P, Mardjetko S. Comparison of radiographic and surface topography measurements in adolescents with idiopathic scoliosis. Open Orthop J. 2012;6:261-5.

22.

Berg S, Tropp HT, Leivseth G. Disc height and motion patterns in the lumbar spine in patients operated with total disc replacement or fusion for discogenic back pain. Results from a randomized controlled trial. Spine J. 2011;11(11):991-8.

23.

Lawrence BD, Wang J, Arnold PM, Hermsmeyer J, Norvell DC, Brodke DS. Predicting the risk of adjacent segment pathology after lumbar fusion: a systematic review. Spine (Phila Pa 1976). 2012;37(22 Suppl):123-32.

\section{Figures}

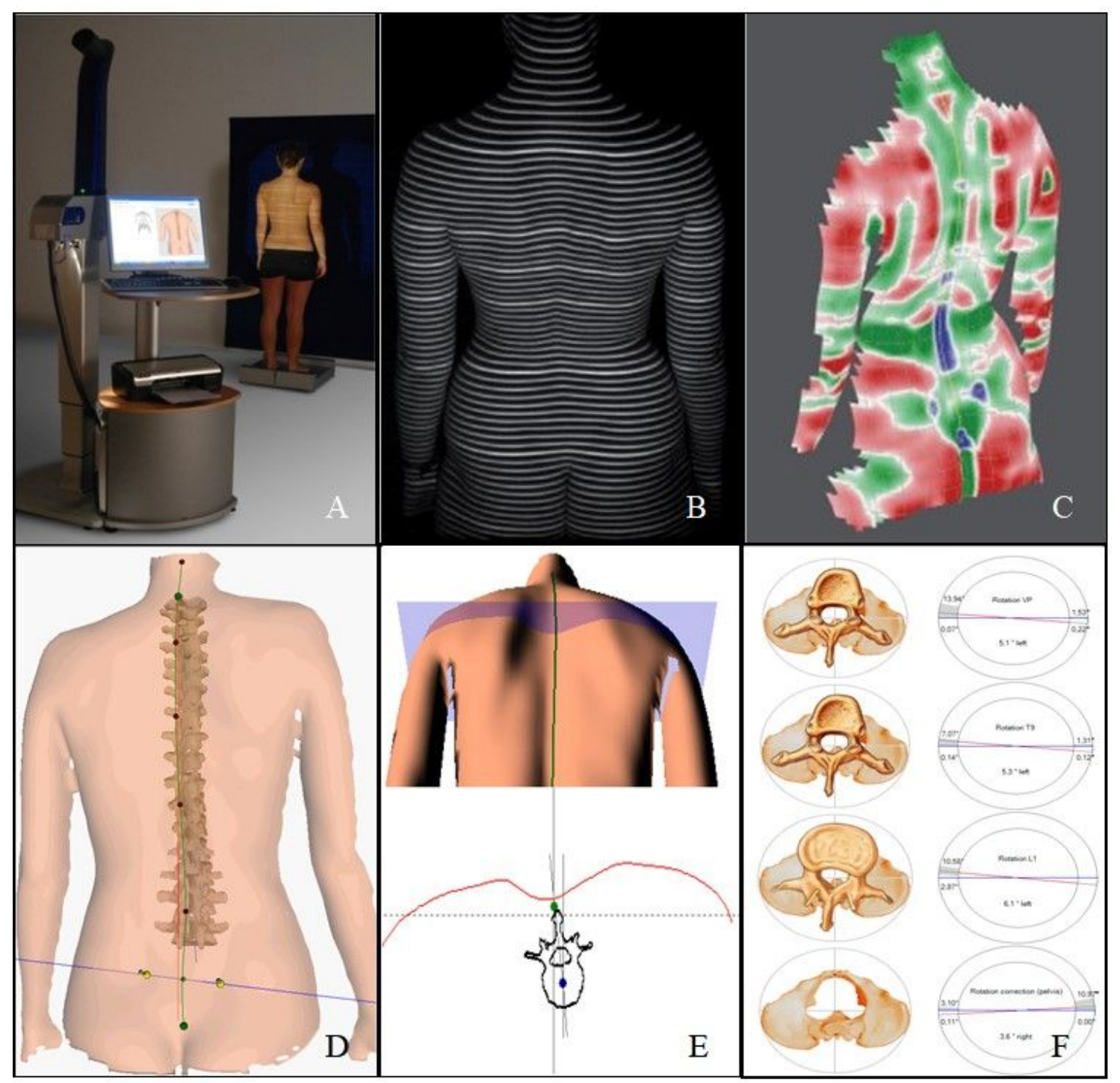

Figure 1

A and B After the undressed (except for the underpants) patient stands on the treadmill, the camera is started to fully capture his/her upper body. The raster lines are then evenly projected on the back; $C$ the patient's anatomy parameters are collected to generate the digital photograph of the back, which is represented as red (convex area), green (saddle area) and blue (concave area); D A three-dimensional spinal model is recreated by the computer. using the collected surface topography data; E and F After the examination and the successful reconstruction, the measurement results during walking is directly available. 


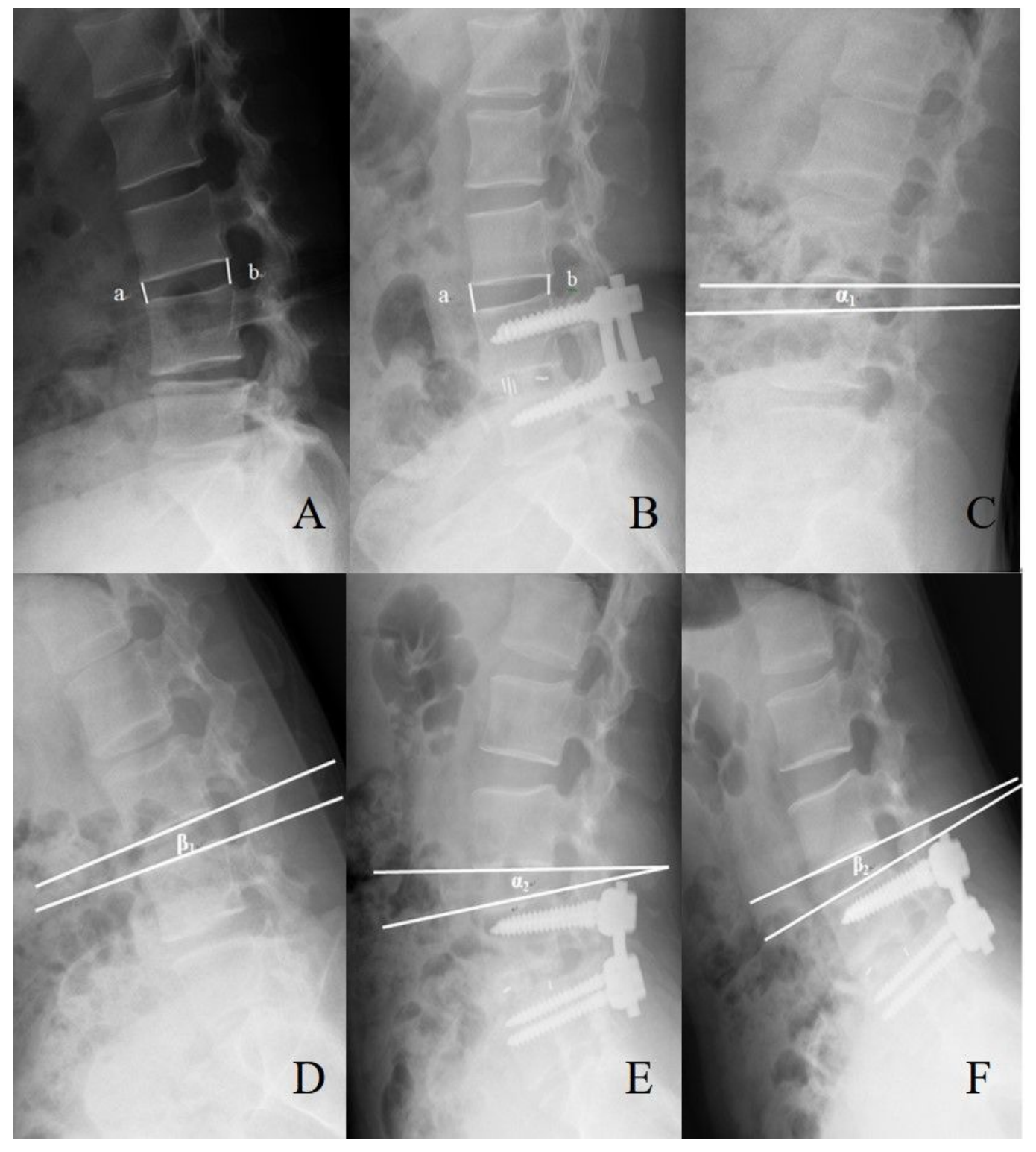

\section{Figure 2}

Adjacent segment disc height $(\mathrm{DH})$ and intervertebral range of montion (ROM) from lateral view lumbar spine $x$-ray. $A$ and $B \mathrm{DH} \otimes(\mathrm{a} \otimes b) / 2$, a and $b$ is measured from the anterior or posterior edges of the upper and lower vertebral bodies respectively; $C \sim F R O M=a \otimes \beta$, $a$ : angulation of the intervertebral space at flexion, $\beta$ : angulation of the intervertebral space at extension. 